\title{
LOCAL CALCULATION OF HOFER'S METRIC AND APPLICATIONS TO BEAM PHYSICS
}

\author{
BELA ERDELYI
}

Communicated by Izu Vaisman

\begin{abstract}
Hofer's metric is a very interesting way of measuring distances between compactly supported Hamiltonian symplectic maps. Unfortunately, it is not known yet how to compute it in general, for example for symplectic maps far away from each other. It is known that Hofer's metric is locally flat, and it can be computed by the so-called oscillation norm of the difference between the Poincare generating functions of symplectic maps close to identity. It is shown here that the same result holds for arbitrary extended generating function types and symplectic maps, as long as the respective generating functions are well defined for the given symplectic maps. This result plays a crucial role is formulating and solving the optimal symplectic approximation problem in Hamiltonian nonlinear dynamics. Applications to beam physics are oulined.
\end{abstract}

\section{Introduction}

Often, in Hamiltonian dynamics, it is necessary to study the quality of the approximation of the real dynamics by some approximate numerical or analytical method, since the equation of the motion cannot be solved analytically in closed form. Lately, the method of choice is the symplectic integration method, which is a category of the so-called geometric integrators [10]. For very complicated weakly nonlinear Hamiltonian systems, as for example large particle accelerators, a variant of the symplectic integration methods is utilized, called symplectification of one-turn maps. To this end, an approximate functional relationship is calculated for trajectories of particles one turn around the accelerator, and the longterm behavior of the beam is obtained by iterating this map. The one-turn map's approximation is not exactly symplectic, and the method that transforms this map into a symplectic map is called symplectification [19]. The study of the symplectification in an optimal way lead to the consideration of Hofer's metric [11] for the formulation of the optimal symplectification conditions [6]. However, the same theory may be applied to symplectic integrators in the traditional sense. The 
success of the optimal symplectification [7,19] underlines the deep connections between the geometric properties of the symplectic diffeomorphisms with respect to Hofer's metric and the dynamics of the corresponding Hamiltonian system that generates the symplectomorphisms.

In this paper, Hofer's metric is reviewed, and a result on the so-called local flatness of Hofer's metric in proved. The local flatness was a crucial argument in the solution of the optimal symplectification [6]. In general, it is not possible to compute Hofer's metric between two arbitrary Hamiltonian symplectic maps. The local flatness phenomenon allows us to do that locally. This local result proved to be sufficient for the optimal symplectification theory. Following a suggestion of McDuff [15], the computation of Hofer's metric between two maps that are close can be done using generating functions (of canonical transformations). In the following sections, we prove that the same formula involving computation of Hofer's metric in terms of generating functions holds for arbitrary generating function types that are valid for the given symplectic maps. In another paper [5], we proved the existence of infinitely many generating functions types. That we can apply the distance computation formula for any type of generating function is the key of the theory of optimal symplectic approximation. More generally, Hofer's metric, the local flatness phenomenon, and related properties may be useful in other aspects of Hamiltonian dynamics.

\subsection{Hofer's Metric}

We use geometric and topological methods to solve a nonlinear dynamics problem. This is astonishing, since despite Hamiltonian systems have been studied for such a long time, there was no symplectic topology 30 years ago. Now symplectic topology is a very lively research field, and we will extend some results concerning Hofer's metric. In [11], a surprising intrinsic metric has been introduced, now called Hofer's metric, on the space of compactly supported Hamiltonian symplectomorphisms, $\operatorname{Ham}^{c}\left(\mathbb{R}^{2 n}\right)$. Recall that a symplectic map is called Hamiltonian if it is the time one map of the flow of some function defined on phase space. The fact that such a Finsler metric exists on a non-compact infinite dimensional Lie group points out the special nature of Hamiltonian systems.

We give a short description of this norm [15]. In general, let $G$ be a Lie group with Lie algebra $L$. A norm $\|\cdot\|$ on $L$ is called invariant if it is invariant under the adjoint action of $G$

$$
\|\xi\|=\left\|g^{-1} \xi g\right\|
$$

(defined through the exponential of $\xi$ at $t=0$ ) for every $\xi \in L$ and every $g \in G$. 
Any such norm gives rise to a bi-invariant intrinsic metric on the Lie group via

$$
d\left(g_{0}, g_{1}\right)=\inf _{g} \int_{0}^{1}\left\|\dot{g}(t) g(t)^{-1}\right\| \mathrm{d} t
$$

for $g_{0}, g_{1} \in G$. The infimum is taken over any smooth path $g:[0,1] \rightarrow G$ connecting $g_{0}=g(0)$ to $g_{1}=g(1)$.

Specifically, the compactly supported Hamiltonian vector fields of $\mathbb{R}^{2 n}$ can be identified with the space of compactly supported functions $C_{c}^{\infty}\left(\mathbb{R}^{2 n}\right)$. The velocity vector of (2) is the Hamiltonian vector field. Hence, Hofer defined the following $L^{\infty}$-type norm on compactly supported Hamiltonian functions

$$
\left\|X_{H_{t}}\right\| \triangleq \sup _{z \in \mathbb{R}^{2 n}} H_{t}(z)-\inf _{z \in \mathbb{R}^{2 n}} H_{t}(z) .
$$

For convenience we denote it as $\left\|H_{t}\right\|$. This norm is called the oscillation norm. The adjoint actions are the symplectic variable changes. For the Hamiltonian functions the adjoint actions are the transformations $H \longmapsto H \circ \psi$, for every $H \in C_{c}^{\infty}\left(\mathbb{R}^{2 n}\right)$ and every $\psi \in \operatorname{Symp}\left(\mathbb{R}^{2 n}, \mathbb{J}\right)$. The oscillation norm is obviously invariant under the adjoint action

$$
\left\|H_{t}\right\|=\left\|H_{t} \circ \psi\right\| .
$$

In fact, the norm stays invariant under the larger diffeomorphism group of $\mathbb{R}^{2 n}$. The induced length spectrum for paths $\left\{\phi_{t}\right\}, t \in[0,1]$ in $\operatorname{Ham}^{c}\left(\mathbb{R}^{2 n}\right)$ is given by

$$
\ell\left\{\phi_{t}\right\}=\int_{0}^{1}\left\|H_{t}\right\| \mathrm{d} t
$$

where $H_{t}$ is the, possibly time dependent, generating Hamiltonian. For any two $\varphi, \psi \in \operatorname{Ham}^{c}\left(\mathbb{R}^{2 n}\right)$, the distance between them is defined as

$$
\rho(\varphi, \psi)=\inf _{\phi_{0}=\varphi, \phi_{1}=\psi} \ell\left\{\phi_{t}\right\}=\inf _{\phi_{0}=\varphi, \phi_{1}=\psi} \int_{0}^{1}\left\|H_{t}\right\| \mathrm{d} t .
$$

The infimum is taken over all smooth paths in $\operatorname{Ham}^{c}\left(\mathbb{R}^{2 n}\right)$ from $\varphi$ to $\psi$. The following proposition holds [12]

Proposition 1. For all $\phi, \varphi, \psi \in \operatorname{Ham}^{c}\left(\mathbb{R}^{2 n}\right)$ the following hold

$$
\begin{aligned}
& \text { - } \rho(\phi, \varphi) \geq 0 \\
& \text { - } \rho(\phi, \varphi)=\rho(\varphi, \phi)
\end{aligned}
$$


- $\rho(\phi, \psi) \leq \rho(\phi, \varphi)+\rho(\varphi, \psi)$

- $\rho(\phi, \mathcal{I})=\rho\left(\varphi \circ \phi \circ \varphi^{-1}, \mathcal{I}\right)$

- $\rho(\psi \circ \phi, \psi \circ \varphi)=\rho(\phi, \varphi)=\rho(\phi \circ \psi, \varphi \circ \psi)$

- the map $t \rightarrow \rho\left(\phi_{t}, \mathcal{I}\right)$ is uniformly continuous.

The first three properties mean that $\rho$ is a pseudo-metric. The highly nontrivial fact is [12]:

Theorem 2. Let $\phi, \varphi \in \operatorname{Ham}^{c}\left(\mathbb{R}^{2 n}\right)$. Then

$$
\rho(\phi, \varphi)=0 \quad \Leftrightarrow \quad \phi=\varphi .
$$

Therefore, Hofer's metric $\rho$ is an essentially unique, genuine, intrinsic, bi-invariant, Finsler metric, i.e., it satisfies the positive definiteness, separation and symmetry axioms, the triangle inequality, and the fifth statement of the above proposition. It has been shown in [4] that all the invariant $L_{p}$ norms, $1 \leq p<\infty$,

$$
\|H\|_{p}=\left(\int_{\mathbb{R}^{\not \ltimes}}|H|^{p} \omega^{n}\right)^{1 / p}
$$

give rise to pseudo-metrics, but not genuine metrics. So, the only non-trivial case is $p=\infty$. We also mention that varying the metric in the $t$ direction gives equivalent metrics.

\subsection{Connectedness of the Group of Hamiltonian Symplectic Maps}

There is a lot of information scattered in the literature on the connectedness of the group of symplectic and Hamiltonian maps $[1,12,15]$. There is also some confusion regarding certain aspects of this topic. For self consistency, the most important results are presented in this subsection.

It follows from the existence of generating functions (or the so-called Weinstein charts) that the group of symplectic maps (compactly supported symplectic maps) are locally contractible, and consequently are locally connected by smooth arcs. Then, the identity components consist of all symplectic maps which are isotopic to the identity through symplectic maps (compactly supported symplectic maps). Smooth isotopies are in one-to-one correspondence with families of smooth vector fields, i.e., if $\phi_{t}$ is such an isotopy, then it gives rise to the vector fields

$$
X_{t}=\frac{\mathrm{d} \phi_{t}}{\mathrm{~d} t} \circ \phi_{t}^{-1} \text {. }
$$


If the isotopy is symplectic, then so is the vector field. On simply connected manifolds, the symplectic vector fields are Hamiltonian (in general, on connected manifolds, only locally Hamiltonian). Therefore, on $\mathbb{R}^{2 n}$ every symplectic isotopy is Hamiltonian. Moreover, it can be shown that in this case the group of symplectic and Hamiltonian maps coincide. Indeed, given any $\mathcal{M} \in \operatorname{Symp}\left(\mathbb{R}^{2 n}\right)$, to show that it is Hamiltonian, according to the above discussion, it is enough to show that $\mathcal{M}$ is the endpoint of a symplectic isotopy.

Denote the constant part of $\mathcal{M}$ by $c$, i.e., $\mathcal{M}(0)=c$. Homotop $\mathcal{M}=c+\mathcal{M}^{0}$ to an origin preserving symplectic map by $\mathcal{M}_{t}=t c+\mathcal{M}^{0}, t \in[0,1]$. We have that $\mathcal{M}_{t}$ is symplectic for each $t$ and $\mathcal{M}^{0}(0)=0$. Using the Alexander trick, an arc can be found from the linear part of $\mathcal{M}^{0}, \mathcal{L}$, to $\mathcal{M}^{0}$, that is

$$
\mathcal{M}_{t}^{0}=\frac{1}{t} \mathcal{M}^{0} \circ t \mathcal{I}
$$

for $t \in(0,1]$. It is easy to see that $\mathcal{M}_{t}^{0}$ is symplectic for each $t$ (the Alexander trick being in fact only a change of scale), and from the Taylor expansion of $\mathcal{M}_{t}^{0}$ is follows that

$$
\lim _{t \rightarrow 0} \mathcal{M}_{t}^{0}=\mathcal{L} .
$$

Furthermore, $L=\operatorname{Jac}(\mathcal{L}) \in \operatorname{Sp}(2 n, \mathbb{R})$, and it is well-known that $\operatorname{Sp}(2 n, \mathbb{R})$ is contractible. There are several ways to see this. For example, any $L \in \operatorname{Sp}(2 n, \mathbb{R})$ can be written as

$$
L=\mathrm{e}^{J S_{1}} \mathrm{e}^{J S_{2}}
$$

where $S_{1}, S_{2}$ are symmetric matrices and $J$ is the matrix of the standard symplectic structure [3]. Therefore, it is enough to define the arc

$$
\mathcal{L}_{t}=\mathrm{e}^{t \mathcal{J} \mathcal{S}_{1}} \circ \mathrm{e}^{t \mathcal{J} \mathcal{S}_{2}}
$$

$t \in[0,1]$ to obtain the final part of the total isotopy. Putting together the different parts by juxtaposition of paths, we obtain the following piecewise smooth isotopy

$$
\mathcal{M}_{t}=\left\{\begin{array}{l}
\mathcal{L}_{t}, t \in[0,1 / 3] \\
\mathcal{M}_{t}^{0}, t \in(1 / 3,2 / 3] \\
t c+\mathcal{M}^{0}, t \in(2 / 3,1] .
\end{array}\right.
$$

By replacing the parameter $t$ by a smooth function $f:[0.1] \rightarrow[0,1]$ such that it is constant in the neighborhoods of the non-smooth points, we finally obtain a smooth isotopy from the identity to $\mathcal{M}$, showing that every $\mathcal{M} \in \operatorname{Symp}\left(\mathbb{R}^{2 n}\right)$ is in fact $\in \operatorname{Ham}\left(\mathbb{R}^{2 n}\right)$. 
The next natural question is the following: if $\mathcal{M} \in \operatorname{Symp}^{c}\left(\mathbb{R}^{2 n}\right)$, then is it true that $\mathcal{M} \in \operatorname{Ham}^{c}\left(\mathbb{R}^{2 n}\right)$ ? In general, the answer is not known. It is true for $n<3$ [9]. Moreover, it is known that $\operatorname{Ham}^{c}\left(\mathbb{R}^{2 n}\right)$ is the $C^{0}$ closure of $\operatorname{Symp}^{c}\left(\mathbb{R}^{2 n}\right)$ for any $n$. Again, there are several ways to see this. Perhaps the easiest is to notice that for any compactly supported diffeomorphism $\phi$ such that $\operatorname{supp}(\phi) \subset$ $D$, and any diffeomorphism $\theta, \operatorname{supp}\left(\theta \circ \phi \circ \theta^{-1}\right) \subset \theta(D)$. It follows that any $\mathcal{M} \in \operatorname{Symp}^{c}\left(\mathbb{R}^{2 n}\right)$ can be conformally rescaled to have support in an arbitrary small neighborhood of a point. Then, the Alexander trick (10) gives an isotopy from $\mathcal{M}$ to an element in $\operatorname{Symp}^{c}\left(\mathbb{R}^{2 n}\right)$ arbitrarily $C^{0}$-close to the identity. Therefore, for computational purposes we can always interchange symplectic with Hamiltonian, even in the compactly supported case.

\section{Local Flatness of Hofer's Metric}

Parametrization of a neighborhood of $\mathcal{M}$ is possible in the $C^{1}$ topology, utilizing the theory of generating functions. The first results in this direction have been obtained in [2] for Hamiltonian maps $C^{1}$ close to identity and Poincaré's generating function, and then it was extended to Hamiltonian maps $C^{1}$ close to identity and all compactly supported generating functions in [13] and [15]. While the approach of [13] is more general, as it holds on any symplectic manifold, we are only interested in $\mathbb{R}^{2 n}$, and the method of [2] lends itself more easily to generalizations. The main idea is to replace the Hamiltonian maps by their generating functions, and try to express Hofer's metric between two maps as some norm of the difference of their generating functions. In [2] this was proven to be possible in some cases. However, in the extended theory of generating functions $[5,7]$ it was shown that in fact there exist uncountably many generator types for any symplectic map, some of which are not compactly supported. To be able to decide which generating function type provides the optimal symplectification, the result of [2] must be generalized to every generator type.

More precisely, introducing a map $\Phi_{\alpha}$ that sends a symplectic map $\mathcal{M}$ into its generating function $F$ of type $\alpha$, we prove that $\Phi_{\alpha}$ is an isometry. Formally, we can state the result as the following main theorem:

Theorem 3. There exists a neighborhood $\mathcal{E}$ of any $\mathcal{M} \in \operatorname{Ham}^{c}\left(\mathbb{R}^{2 n}\right)$, and a neighborhood $\mathcal{Z}$ of 0 in $C_{c}^{\infty}\left(\mathbb{R}^{2 n}\right)$ such that the map

$$
\Phi_{\alpha}: \mathcal{E} \rightarrow \mathcal{Z}, \quad \Phi_{\alpha}(\mathcal{M})=F
$$


is isometric. That is, for every $F, G \in \mathcal{Z}$

$$
\rho(\mathcal{M}, \mathcal{N})=\frac{1}{|\mu|}\left\|\Phi_{\alpha}(\mathcal{M})-\Phi_{\alpha}(\mathcal{N})\right\|=\frac{1}{|\mu|}\|F-G\| .
$$

As a consequence, the inverse of the isometry takes any function from $(\mathcal{Z},\|\cdot\|)$ into a Hamiltonian symplectomorphism in $(\mathcal{E}, \rho)$ depending on $\alpha$. This shows that, considering the space $(\mathcal{Z},\|\cdot\|)$ a flat space (in which straight lines are minimal geodesics), their image under $\Phi_{\alpha}^{-1}: \mathcal{Z} \rightarrow \mathcal{E}$ in $\operatorname{Ham}^{c}\left(\mathbb{R}^{2 n}\right)$ remain locally flat minimal geodesics. That is why this results is called the local flatness phenomenon.

The proof uses three main ingredients. First, there is an intimate relationship between fixed points of symplectic maps and critical points of generating functions, which is presented in the next subsection. Second, the proof in [2] is based on the Hamilton-Jacobi equation. In the following the generalized Hamilton-Jacobi equation is derived, adapted to our situation. It provides the time evolution of any generator type. Finally, the proof uses a theorem of Siburg [20], which is stated without proof. The theorem essentially states that paths without nontrivial fixed points are absolutely length minimizing for Hofer's metric.

\subsection{Review of the Extended Generating Function Theory}

Here we summarize the main result of the extended generating function theory $[5,7]$.

Definition 4. A function $F$ defined by

$$
(\nabla F)^{T}=\left(\alpha_{1} \circ\left(\begin{array}{c}
\mathcal{M} \\
\mathcal{I}
\end{array}\right)\right) \circ\left(\alpha_{2} \circ\left(\begin{array}{c}
\mathcal{M} \\
\mathcal{I}
\end{array}\right)\right)^{-1}
$$

where $\mathcal{M}$ is a symplectic map and $\alpha=\left(\alpha_{1}, \alpha_{2}\right)^{T}$ is a conformal symplectic map satisfying

$$
(\operatorname{Jac}(\alpha))^{T}\left(\begin{array}{cc}
0_{2 n} & I_{2 n} \\
-I_{2 n} & 0_{2 n}
\end{array}\right)(\operatorname{Jac}(\alpha))=\mu\left(\begin{array}{cc}
J_{2 n} & 0_{2 n} \\
0_{2 n} & -J_{2 n}
\end{array}\right)
$$

$\mu \in \mathbb{R}^{\times}$, is called a generating function of type $\alpha$ for $\mathcal{M}$.

Of course, the existence is tied to the existence of the inverse in the defining relation, but always there are infinitely many conformal symplectic maps that satisfy 
this requirement for any given symplectic map, so it follows that to any symplectic map infinitely many generating function types can be constructed.

Also, it follows from the above definition that the inverse relationship

$$
\mathcal{M}=\left(\alpha^{1} \circ\left(\begin{array}{c}
\nabla F \\
\mathcal{I}
\end{array}\right)\right) \circ\left(\alpha^{2} \circ\left(\begin{array}{c}
\nabla F \\
\mathcal{I}
\end{array}\right)\right)^{-1}
$$

holds with $\alpha^{-1}=\left(\alpha^{1}, \alpha^{2}\right)^{T}$.

\subsection{The Fixed Point-Critical Point Relationship}

In this subsection we study an interesting property of generating functions. A point $z_{f}$ is called a fixed point of the symplectic map $\mathcal{M}$ if it acts on it as the identity map, that is $\mathcal{M}\left(z_{f}\right)=z_{f}$. It follows that the iterates of the map have the same fixed points,

$$
\mathcal{M}^{k}\left(z_{f}\right)=\mathcal{M}^{k-1} \circ \mathcal{M}\left(z_{f}\right)=\mathcal{M}^{k-1}\left(z_{f}\right)=\ldots=\mathcal{M}\left(z_{f}\right)=z_{f} .
$$

Moreover, the inverse $\mathcal{M}^{-1}$ also has the same fixed points,

$$
\mathcal{M}^{-1}\left(z_{f}\right)=\mathcal{M}^{-1} \circ \mathcal{M}\left(z_{f}\right)=z_{f} .
$$

To sum up, we can say that any integer power of a symplectic map has the same fixed points as the map itself. Thus the set of fixed points form a topological invariant of the map under iteration. Generating functions can be connected with these fixed points. For start, let us consider a special class of generating functions. We assume that the generating functions are globally defined, otherwise the theory is valid for the fixed points in the regions where they are defined. The critical points of functions in this set are the fixed points of the symplectic maps, and conversely, the fixed points of the map are critical points of the function. If the symplectic maps are compactly supported, then these generating functions are exactly the generating functions with compact support. This can be easily seen if we argue geometrically. Recall that a symplectic map is a Lagrangian submanifold in the product manifold. Then, the fixed points of the map are exactly the intersection points of this submanifold with the diagonal. On the other hand, the critical points of the generating function are the intersection points of the Lagrangian submanifold determined by the function with the zero section. Now, if we identify the diagonal with the zero section by some given $\alpha$, the fixed points of the map will go into critical points of the generating functions. 
Explicitly, given a symplectic map, we can choose only $\alpha$ 's that satisfy the transversality condition. On the other hand, identification of the diagonal with the zero section requires that

$$
\alpha(\Delta)=Z
$$

which further restricts the pool of $\alpha$ 's. A necessary condition is that the generating functions in this class can generate the identity map. If the map is close enough to the identity, then also the transversality condition is satisfied automatically. Equation (22) can be expanded as

$$
\left(\begin{array}{c}
\alpha_{1} \circ\left(\begin{array}{l}
\mathcal{I} \\
\mathcal{I} \\
\mathcal{I} \\
\alpha_{2} \circ
\end{array}\right) \\
\mathcal{I}
\end{array}\right)(z)=\left(\begin{array}{l}
0 \\
\mathcal{I}
\end{array}\right)(w)
$$

Applying $z_{f}$ to (17), we obtain that

$$
\nabla F \circ \alpha_{2} \circ\left(\begin{array}{c}
\mathcal{M} \\
\mathcal{I}
\end{array}\right)\left(z_{f}\right)=\alpha_{1} \circ\left(\begin{array}{c}
\mathcal{M} \\
\mathcal{I}
\end{array}\right)\left(z_{f}\right)
$$

which is equivalent to

$$
\nabla F \circ \alpha_{2} \circ\left(\begin{array}{l}
\mathcal{I} \\
\mathcal{I}
\end{array}\right)\left(z_{f}\right)=\alpha_{1} \circ\left(\begin{array}{l}
\mathcal{I} \\
\mathcal{I}
\end{array}\right)\left(z_{f}\right)
$$

Using (23) we arrive to

$$
\nabla F\left(w_{f}\right)=0
$$

where $w_{f}$ is given by $z_{f}$ and the identification process. From the explicit constraints we will see that actually $w_{f}=z_{f}$. Hence, the fixed points of the map are critical points of the generating functions from this set.

The next question is whether this is true conversely: are all the critical points of generating functions in this set fixed points of the map? As has been shown,

$$
\mathcal{M}=\left(\alpha^{1} \circ\left(\begin{array}{c}
\nabla F \\
\mathcal{I}
\end{array}\right)\right) \circ\left(\alpha^{2} \circ\left(\begin{array}{c}
\nabla F \\
\mathcal{I}
\end{array}\right)\right)^{-1}
$$

or

$$
\mathcal{M} \circ\left(\alpha^{2} \circ\left(\begin{array}{c}
\nabla F \\
\mathcal{I}
\end{array}\right)\right)\left(w_{c}\right)=\left(\alpha^{1} \circ\left(\begin{array}{c}
\nabla F \\
\mathcal{I}
\end{array}\right)\right)\left(w_{c}\right)
$$


where $w_{c}$ are the critical points of $F$. From (22) it follows that

$$
\begin{gathered}
\alpha^{-1}(Z)=\Delta \\
\left(\begin{array}{c}
\alpha^{1} \circ\left(\begin{array}{l}
0 \\
\mathcal{I}
\end{array}\right) \\
\alpha^{2} \circ\left(\begin{array}{l}
0 \\
\mathcal{I}
\end{array}\right)
\end{array}\right)(w)=\left(\begin{array}{c}
\mathcal{I} \\
\mathcal{I}
\end{array}\right)(z) .
\end{gathered}
$$

Combining what we have derived sofar, we are able to answer the question about critical points. Using $\nabla F\left(w_{c}\right)=0$, from (28) and (30) we obtain

$$
\mathcal{M}\left(z_{c}\right)=z_{c}
$$

Again, $z_{c}$ is the point corresponding to $w_{c}$ via the identification, and we will see that $z_{c}=w_{c}$. Hence, $w_{c}$ are fixed points of the symplectic map. In conclusion, there is a one-to-one correspondence between fixed points of the map and critical points of the generating functions in this set.

Now we elaborate in the direction of finding the explicit constraints for this class of generating functions. Beside the constraints given by (18) we have another set given by (23), which in terms of the entries in the Jacobian of $\alpha$ read

$$
\begin{aligned}
& A(z, z)+B(z, z)=0 \\
& C(z, z)+D(z, z)=I .
\end{aligned}
$$

But we already know that [7], with $M=I$, this set is nothing else than generating functions constructed via any $\alpha$ of the form

$$
\alpha=\left(\begin{array}{cc}
-\mu J & \mu J \\
\frac{1}{2}(I+J S) & \frac{1}{2}(I-J S)
\end{array}\right) \quad \mu \in \mathbb{R}^{\times}, \quad S=S^{T} .
$$

The critical points of these generators are in one-to-one correspondence with the fixed points of the map. Now it is straightforward to check that for any generating function in this set indeed $w_{f}=z_{f}$ and $z_{c}=w_{c}$.

The above results can be extended to any generating function, with the condition that it can be used to generate the identity map. The difference between the above set and the other generating functions is that they will not identify the diagonal with the zero section, but some other section. However, this little inconvenience can be circumvented by noticing that subtracting from the generating function the function that generates the identity map, we get almost the same results. That is, 
suppose we have the following

$$
\begin{aligned}
& \nabla F \circ \alpha_{2} \circ\left(\begin{array}{c}
\mathcal{M} \\
\mathcal{I}
\end{array}\right)(z)=\alpha_{1} \circ\left(\begin{array}{c}
\mathcal{M} \\
\mathcal{I}
\end{array}\right)(z) \\
& \nabla F_{0} \circ \alpha_{2} \circ\left(\begin{array}{c}
\mathcal{I} \\
\mathcal{I}
\end{array}\right)(z)=\alpha_{1} \circ\left(\begin{array}{c}
\mathcal{I} \\
\mathcal{I}
\end{array}\right)(z)
\end{aligned}
$$

where we denoted by $F_{0}$ the same type of generating function as $F$, but which generates the identity map. Then, if $z=z_{f}$, the right hand sides are the same, and we obtain that

$$
\nabla\left(F-F_{0}\right)\left(w_{f}\right)=0 .
$$

The fixed points can be obtained from

$$
z_{f}=\left(\alpha_{2} \circ\left(\begin{array}{l}
\mathcal{I} \\
\mathcal{I}
\end{array}\right)\right)^{-1}\left(w_{f}\right) .
$$

So, there is still a one-to-one correspondence between fixed points of symplectic maps and critical points of generating functions in the above modified sense. Of course, in the case of the (34) set $F_{0}=0$, and the previous results can be recovered.

Finally, to put the results in the form that we will need them to prove the theorem, consider the case when the same generator type $\alpha$ exists for two symplectic maps $\mathcal{M}$ and $\mathcal{N}$. It follows that

$$
\begin{gathered}
\nabla F \circ \alpha_{2} \circ\left(\begin{array}{c}
\mathcal{M} \\
\mathcal{I}
\end{array}\right)(z)=\alpha_{1} \circ\left(\begin{array}{c}
\mathcal{M} \\
\mathcal{I}
\end{array}\right)(z) \\
\nabla G \circ \alpha_{2} \circ\left(\begin{array}{c}
\mathcal{N} \\
\mathcal{I}
\end{array}\right)(z)=\alpha_{1} \circ\left(\begin{array}{c}
\mathcal{N} \\
\mathcal{I}
\end{array}\right)(z) .
\end{gathered}
$$

Then, on the set of common fixed points $z_{f}$ of $\mathcal{M}$ and $\mathcal{N}$ we obtain that

$$
\nabla(F-G)\left(w_{f}\right)=0 .
$$

\subsection{The Generalized Hamilton-Jacobi Equation and Applications}

In this subsection we prove the most general Hamilton-Jacobi equation, associated to the time evolution of any generating function. As we have seen, critical points of generating functions are closely related to fixed points of the map. But more can be said. We will show that, actually every generating function of compact support assumes the same value at the fixed points of the map. These numbers 
are called the action of the fixed points, that is, if $z_{f}$ is a fixed point of $\mathcal{M}$, define $\mathcal{A}\left(z_{f}, \mathcal{M}, \mu\right)$ as the value taken by any generating function. Call the set of all such numbers, as $z_{f}$ spans all the fixed points, the action spectrum of $\mathcal{M}$

$$
\sigma_{\mu}(\mathcal{M})=\left\{\mathcal{A}\left(z_{f}, \mathcal{M}, \mu\right) ; \mathcal{M}\left(z_{f}\right)=z_{f}\right\} .
$$

The spectrum depends parametrically on the conformality factor $\mu$ of $\alpha$, and is a symplectic invariant of the map. To show the claim, we need to prove that the generating functions satisfy a generalized Hamilton-Jacobi equation. Based on the proof in [8] for linear $\alpha$ 's, here we present the general proof. Assume that the flow is generated by the time-dependent Hamiltonian $H_{t}$, and the corresponding time $t$ maps can be represented at any moment by a time-dependent generating function $F_{t}$. We claim the following.

Theorem 5. The following Hamilton-Jacobi equation is satisfied for any $w \in$ $\mathbb{R}^{2 n}$ :

$$
\frac{\partial}{\partial t} F_{t}(w)=\mu H_{t} \circ\left(\alpha^{1} \circ\left(\begin{array}{c}
\mathcal{N}_{t} \\
\mathcal{I}
\end{array}\right)\right)(w)
$$

where $F_{t}$ is the generating function associated to any $\alpha$ satisfying (18), and $\mathcal{N}_{t}=$ $\left(\nabla F_{t}\right)^{T}$ at every moment $t$.

Proof: We are situated in the $\left(\mathbb{R}^{4 n}, \mathbb{J}_{4 n}\right)$ symplectic space, with symplectic coordinates $(w, \hat{w})$. Consider the extended phase space $\mathbb{R}^{4 n+2}$ by including the canonical pair of variables $\left(t,-\bar{H}_{t}\right)$. The symplectic structure modifies to

$$
\omega=\omega_{0}-\mathrm{d} t \wedge \mathrm{d} \bar{H}_{t}
$$

the differential form of Cartan. Take the one-form

$$
\lambda_{e}=\hat{w} \mathrm{~d} w+\bar{H}_{t} \mathrm{~d} t
$$

where $\bar{H}_{t}=\mu H_{t} \circ\left(\alpha^{1} \circ\left(\begin{array}{c}\mathcal{N}_{t} \\ \mathcal{I}\end{array}\right)\right)$. We prove that $\lambda_{e}$ is closed. We have that

$$
\begin{aligned}
\mathrm{d} \lambda_{e} & =\sum_{i, j=1}^{2 n} \frac{\partial \hat{w}_{i}}{\partial w_{j}} \mathrm{~d} w_{j} \wedge \mathrm{d} w_{i}+\sum_{i=1}^{2 n}\left(\frac{\partial \hat{w}_{i}}{\partial t} \mathrm{~d} t \wedge \mathrm{d} w_{i}+\frac{\partial \bar{H}_{t}}{\partial w_{i}} \mathrm{~d} w_{i} \wedge \mathrm{d} t\right)+\frac{\partial \bar{H}_{t}}{\partial t} \mathrm{~d} t \wedge \mathrm{d} t \\
& =\sum_{i<j}^{2 n}\left(\operatorname{Jac}\left(\mathcal{N}_{t}\right)-\operatorname{Jac}\left(\mathcal{N}_{t}\right)^{T}\right) \mathrm{d} w_{j} \wedge \mathrm{d} w_{i}+\sum_{i=1}^{2 n}\left(\frac{\partial \hat{w}_{i}}{\partial t}-\frac{\partial \bar{H}_{t}}{\partial w_{i}}\right) \mathrm{d} t \wedge \mathrm{d} w_{i} \\
& =\sum_{i=1}^{2 n}\left(\frac{\partial \hat{w}_{i}}{\partial t}-\frac{\partial \bar{H}_{t}}{\partial w_{i}}\right) \mathrm{d} t \wedge \mathrm{d} w_{i} .
\end{aligned}
$$


On the other hand

$$
\begin{aligned}
& \frac{\mathrm{d} \hat{w}}{\mathrm{~d} t}=\dot{\mathcal{N}}_{t}(w)=\operatorname{Jac}\left(\mathcal{N}_{t}(w)\right) \cdot \dot{w}+\frac{\partial \hat{w}}{\partial t} \\
& \frac{\partial \hat{w}}{\partial t}=\dot{\mathcal{N}}_{t}(w)-\operatorname{Jac}\left(\mathcal{N}_{t}(w)\right) \cdot \dot{w} .
\end{aligned}
$$

Recall that

$$
\begin{aligned}
& w=\alpha_{2} \circ\left(\begin{array}{c}
\mathcal{M}_{t} \\
\mathcal{I}
\end{array}\right)(z) \\
& \hat{w}=\alpha_{1} \circ\left(\begin{array}{c}
\mathcal{M}_{t} \\
\mathcal{I}
\end{array}\right)(z)
\end{aligned}
$$

and $\hat{z}=\mathcal{M}_{t}(z)$ is the solution of the initial value problem

$$
\begin{aligned}
\frac{\mathrm{d} \hat{z}}{\mathrm{~d} t} & =J \nabla_{\hat{z}} H_{t}(\hat{z}) \\
\hat{z}(t=0) & =z .
\end{aligned}
$$

We compute

$$
\begin{aligned}
& \frac{\partial \bar{H}_{t}}{\partial w}(w)=\mu \nabla_{w}\left(H_{t} \circ\left(\alpha^{1} \circ\left(\begin{array}{c}
\mathcal{N}_{t} \\
\mathcal{I}
\end{array}\right)\right)\right)(w) \\
& =\mu\left[\operatorname{Jac}\left(\alpha^{1} \circ\left(\begin{array}{c}
\mathcal{N}_{t} \\
\mathcal{I}
\end{array}\right)\right)(w)\right]^{T} \cdot \nabla_{\hat{z}} H(\hat{z}) \\
& =\mu\left[\left(\begin{array}{cc}
A^{\alpha} & B^{\alpha}
\end{array}\right) \cdot\left(\begin{array}{c}
N_{t} \\
I
\end{array}\right)\right]^{T} \cdot \nabla_{\hat{z}} H(\hat{z}) \\
& =\mu\left(A^{\alpha} N_{t}+B^{\alpha}\right)^{T} \cdot \nabla_{\hat{z}} H(\hat{z}) \\
& =\mu\left(N_{t} A^{\alpha T}+B^{\alpha T}\right) \cdot \nabla_{\hat{z}} H(\hat{z})
\end{aligned}
$$

where in the second row we used $\hat{z}=\alpha^{1} \circ\left(\begin{array}{c}\mathcal{N}_{t} \\ \mathcal{I}\end{array}\right)(w)$, in the third row $\operatorname{Jac}\left(\mathcal{N}_{t}\right)=N_{t}$ and the notation for the Jacobian of $\alpha^{-1}$

$$
\alpha^{\#}=\operatorname{Jac}\left(\alpha^{-1}\right)=\left(\begin{array}{ll}
A^{\alpha} & B^{\alpha} \\
C^{\alpha} & D^{\alpha}
\end{array}\right) .
$$

In the last row the fact that $N_{t}$ is symmetric, $N_{t}^{T}=N_{t}$ is also utilized. We can express $\alpha^{\#}$ in terms of entries of $\alpha_{\#}$. From (18) it follows that

$$
\begin{aligned}
\alpha^{\#} & =-\frac{1}{\mu} \tilde{J}_{4 n} \alpha_{\#}^{T} J_{4 n} \\
& =-\frac{1}{\mu}\left(\begin{array}{cc}
J_{2 n} & 0_{2 n} \\
0_{2 n} & -J_{2 n}
\end{array}\right)\left(\begin{array}{cc}
A^{T} & C^{T} \\
B^{T} & D^{T}
\end{array}\right)\left(\begin{array}{cc}
0_{2 n} & I_{2 n} \\
-I_{2 n} & 0_{2 n}
\end{array}\right)
\end{aligned}
$$


which reads explicitly

$$
\begin{aligned}
A^{\alpha} & =\frac{1}{\mu} J_{2 n} C^{T}, & B^{\alpha} & =-\frac{1}{\mu} J_{2 n} A^{T} \\
C^{\alpha} & =-\frac{1}{\mu} J_{2 n} D^{T}, & D^{\alpha} & =\frac{1}{\mu} J_{2 n} B^{T} .
\end{aligned}
$$

Finally, we obtain

$$
\begin{aligned}
\frac{\partial \bar{H}_{t}}{\partial w}(w) & =\mu\left(N_{t} A^{\alpha T}+B^{\alpha T}\right) \cdot \nabla_{\hat{z}} H_{t}(\hat{z}) \\
& =\left(A-N_{t} C\right) \cdot J \nabla_{\hat{z}} H_{t}(\hat{z}) .
\end{aligned}
$$

Analogously, the calculation of $\frac{\partial \hat{w}}{\partial t}$ proceeds as follows

$$
\begin{aligned}
\frac{\partial \hat{w}}{\partial t} & =\frac{\mathrm{d}}{\mathrm{d} t}\left(\alpha_{1}(\hat{z}, z)\right)-N_{t} \cdot \frac{\mathrm{d}}{\mathrm{d} t}\left(\alpha_{2}(\hat{z}, z)\right) \\
& =\frac{\partial \alpha_{1}(\hat{z}, z)}{\partial \hat{z}} \cdot \frac{\mathrm{d} \hat{z}}{\mathrm{~d} t}-N_{t} \cdot \frac{\partial \alpha_{2}(\hat{z}, z)}{\partial \hat{z}} \cdot \frac{\mathrm{d} \hat{z}}{\mathrm{~d} t} \\
& =\left(A-N_{t} C\right) \cdot J \nabla_{\hat{z}} H_{t}(\hat{z}) .
\end{aligned}
$$

Hence, $\mathrm{d} \lambda_{e}=0$. The vanishing first cohomology class guarantees the existence of a function $F_{t}(w)$ such that

$$
\begin{aligned}
\mathrm{d} F_{t}(w) & =\lambda_{e}=\hat{w} \mathrm{~d} w+\bar{H}_{t}(w) \mathrm{d} t \\
\frac{\partial F_{t}(w)}{\partial w} \mathrm{~d} w+\frac{\partial F_{t}(w)}{\partial t} \mathrm{~d} t & =\hat{w} \mathrm{~d} w+\bar{H}_{t}(w) \mathrm{d} t .
\end{aligned}
$$

Comparing coefficients, we get that indeed $F_{t}(w)$ is the generating function

$$
\nabla F_{t}(w)=\mathcal{N}_{t}(w)=\hat{w}
$$

and the advertised result

$$
\frac{\partial F_{t}(w)}{\partial t}=\mu H_{t} \circ\left(\alpha^{1} \circ\left(\begin{array}{c}
\mathcal{N}_{t} \\
\mathcal{I}
\end{array}\right)\right)(w) .
$$

Finally, as an application, it can be shown that compactly supported generating functions assume the same value at the fixed points of the symplectic map. Notice that the right hand side of (71) can be expressed as

$$
\begin{aligned}
H_{t} \circ\left(\alpha^{1} \circ\left(\begin{array}{c}
\mathcal{N}_{t} \\
\mathcal{I}
\end{array}\right)\right)(w) & =H_{t} \circ\left(\alpha^{1} \circ \alpha \circ\left(\begin{array}{c}
\mathcal{M}_{t} \\
\mathcal{I}
\end{array}\right)(z)\right) \\
& =H_{t} \circ \mathcal{M}_{t}(z) .
\end{aligned}
$$


In the derivation we used the following

$$
\alpha^{-1} \circ \alpha=\left(\begin{array}{l}
\alpha^{1} \circ \alpha \\
\alpha^{2} \circ \alpha
\end{array}\right)=\mathcal{I} .
$$

Therefore, $\alpha^{1} \circ \alpha$ is the identity for the first $2 n$ components and 0 for the second $2 n$ components. Also, if we consider only time-independent Hamiltonians, $H$ is invariant under its own flow at any time, $H=H \circ \mathcal{M}_{t}$, since $H$ is constant along the solutions of the Hamiltonian dynamical system. Reparametrization with respect to time, such that $\mathcal{M}$ is the time 1 flow of $H_{t}$, and integration of (68) gives

$$
\begin{aligned}
& F \circ \alpha_{2} \circ\left(\begin{array}{c}
\mathcal{M} \\
\mathcal{I}
\end{array}\right)(z)-F_{0} \circ \alpha_{2} \circ\left(\begin{array}{c}
\mathcal{I} \\
\mathcal{I}
\end{array}\right)(z) \\
& =\int_{w(0)}^{w(1)} \nabla F(w) \cdot \mathrm{d} w+\mu \int_{0}^{1} H_{t} \circ \mathcal{M}_{t}(z) \mathrm{d} t .
\end{aligned}
$$

We used $\mathcal{M}_{0}=\mathcal{I}$, and the notation $F_{1}=F$. If $z=z_{f}$, the first term on the right hand side is vanishing because in this case $w(0)=w(1)$ as can be seen from (49). Hence, we get

$$
\left(F-F_{0}\right)\left(w_{f}\right)=\mu \int_{0}^{1} H_{t} \circ \mathcal{M}_{t}\left(z_{f}\right) \mathrm{d} t
$$

with

$$
w_{f}=\alpha_{2} \circ\left(\begin{array}{l}
\mathcal{I} \\
\mathcal{I}
\end{array}\right)\left(z_{f}\right) .
$$

In particular, for compactly supported generating functions (77) takes the form

$$
F\left(z_{f}\right)=\mu \int_{0}^{1} H_{t} \circ \mathcal{M}_{t}\left(z_{f}\right) \mathrm{d} t=\mathcal{A}\left(z_{f}, \mathcal{M}, \mu\right) .
$$

This means that generating functions in this set, with the same conformality factor, take the same value at the fixed points of the symplectic map. In general, the other generating functions, after subtracting the part that generates the identity, take the same value at points that are in one-to-one correspondence with the fixed points. In [15] it is shown that the action of a fixed point is related to the area enclosed by certain loops, thus is no surprise that the action depends parametrically on the conformality factor.

It is also known that the action spectrum of compactly supported Hamiltonian maps is compact and nowhere dense, and in general does not depend continuously with respect to $\mathcal{M}$. For details we refer the reader to [12]. On the other hand, 
Weinstein proved that the fixed points of a perturbed symplectic map are close to the fixed points of the unperturbed map [23]. A related result is due to Viterbo, who proved that any compactly supported symplectic map has infinitely many periodic points inside its support [22].

\subsection{Siburg's Theorem}

Some preparation is needed before the theorem statement.

Definition 6. Let $I \subset \mathbb{R}$ be a connected subset with non-empty interior. A smooth path $\left\{\phi_{t}\right\}: I \rightarrow \operatorname{Ham}^{c}\left(\mathbb{R}^{2 n}\right)$ is called regular if $X_{H_{t}} \neq 0$ for every $t \in I$.

Definition 7. Let $\left\{\phi_{t}\right\}: I \rightarrow \operatorname{Ham}^{c}\left(\mathbb{R}^{2 n}\right)$ be a smooth regular path.

- $\left\{\phi_{t}\right\}$ is called a minimal geodesic iffor all $a, b \in I$, such that $a<b$,

$$
\left.\ell\left\{\phi_{t}\right\}\right|_{[a, b]}=\rho\left(\phi_{a}, \phi_{b}\right)
$$

holds.

- $\left\{\phi_{t}\right\}$ is called a geodesic if for every $t \in I$ there exists a neighborhood $U \subset I$ of $t$ such that $\left.\left\{\phi_{t}\right\}\right|_{U}$ is a minimal geodesic.

The following proposition is proved in [2].

Proposition 8. Let $H:[a, b] \times \mathbb{R}^{2 n} \rightarrow \mathbb{R}$ be a smooth compactly supported function. The following two conditions are equivalent:

- $\int_{a}^{b}\left\|H_{t}\right\| \mathrm{d} t=\left\|\int_{a}^{b} H_{t}(z) \mathrm{d} t\right\|$

- There exist two points $z_{-}, z_{+} \in \mathbb{R}^{2 n}$ such that $\sup H_{t}=H_{t}\left(z_{+}\right)$and $\inf _{z} H_{t}=H_{t}\left(z_{-}\right)$, for all $t \in[a, b]$.

Definition 9. A function $H_{t}(z)$ which satisfies one of the conditions in the proposition above is called quasi-autonomous. Each autonomous path is quasi-autonomous.

We are interested under what conditions the length-minimizing property of a path is achieved. Lalonde and McDuff [13] proved the necessary condition, which holds for any symplectic manifold. 
Theorem 10. A regular path $\left\{\phi_{t}\right\}, t \in I$, in $\operatorname{Ham}^{c}\left(\mathbb{R}^{2 n}\right)$ is a geodesic if and only if its generating Hamiltonian has at least one fixed maximum and one fixed minimum at each moment.

Hence, every geodesic is generated by quasi-autonomous Hamiltonian functions. To prove that a path is length-minimizing is much harder. Fortunately, in $\mathbb{R}^{2 n}$ one can obtain more results than in the general case. First, we need another definition.

Definition 11. A fixed point, $z_{f}$, of a map $\phi_{1} \in \operatorname{Ham}^{c}\left(\mathbb{R}^{2 n}\right)$ is called constant if it is a fixed point of its flow, that is $\phi_{t}\left(z_{f}\right)=z_{f}$, for every $t \in[0,1]$.

We say that a smooth path $\left\{\phi_{t \in[0,1]}\right\}$ starting at the identity has no non-constant fixed points if for any fixed $\tau \in(0,1]$ and $z_{f}$ such that

$$
\phi_{\tau}\left(z_{f}\right)=z_{f} \Rightarrow \phi_{t}\left(z_{f}\right)=z_{f}
$$

for any $t \in[0,1]$. In case $\left\{\phi_{t \in[0,1]}\right\}$ does not start at the identity, (81) should be modified to

$$
\phi_{\tau}\left(z_{f}\right)=\phi_{0}\left(z_{f}\right) \Rightarrow \phi_{t}\left(z_{f}\right)=\phi_{0}\left(z_{f}\right) .
$$

We are ready to state the following theorem [20].

Theorem 12. Any regular path $\left\{\phi_{t \in[0,1]}\right\}$ in $\operatorname{Ham}^{c}\left(\mathbb{R}^{2 n}\right)$ that is generated by a quasi-autonomous Hamiltonian, and has no non-constant fixed points in time less than one, is a minimal geodesic, that is absolutely length-minimizing for Hofer's metric.

It is conjectured that a similar theorem holds for any symplectic manifold. The case of autonomous Hamiltonians has been proved recently [16].

\subsection{Proof of the Main Theorem}

Now we are ready to derive the so-called local flatness phenomenon. We want to measure the distance between two compactly supported Hamiltonian symplectic maps, say $\varphi$ and $\psi$. Suppose that for some type $\alpha$ the two generating functions associated with $\alpha$ for $\varphi$ and $\psi$ are $F_{\alpha}$ and $G_{\alpha}$. Take the convex combination of the two generating functions

$$
S_{t}=(1-t) F_{\alpha}+t G_{\alpha}=F_{\alpha}+t\left(G_{\alpha}-F_{\alpha}\right) .
$$

The corresponding path $\left\{\phi_{t}\right\}$ in $\operatorname{Ham}^{c}\left(\mathbb{R}^{2 n}\right)$ is generated by a Hamiltonian $H_{t}$. The path satisfies the following relations: $\phi_{0}=\varphi$ and $\phi_{1}=\psi$. Noticing that 
$S_{0}=F_{\alpha}$, if $z_{f}$ is a fixed point of $\phi_{\tau}$, for some fixed $\tau \in(0,1]$, according to (41) it follows that

$$
\nabla\left(S_{\tau}-S_{0}\right)\left(w_{f}\right)=\tau \cdot \nabla\left(G_{\alpha}-F_{\alpha}\right)\left(w_{f}\right)=0
$$

that is $w_{f}$ is a critical point of $G_{\alpha}-F_{\alpha}$. Obviously, this implies that $w_{f}$ remains a critical point of $G_{\alpha}-F_{\alpha}$ for any $t \in[0,1]$, which in turn means that $z_{f}$ is a fixed point of $\phi_{t}$ for any $t \in[0,1]$. Indeed, as in (38), it follows that in this case

$$
z_{f}=\left(\alpha_{2} \circ\left(\begin{array}{l}
\varphi \\
\mathcal{I}
\end{array}\right)\right)^{-1}\left(w_{f}\right) .
$$

We just proved that all the fixed points of $\left\{\phi_{t \in[0,1]}\right\}$ are constant. Also, a careful look at the Hamilton-Jacobi equation (71) reveals that the path $\left\{\phi_{t \in[0,1]}\right\}$ is generated by quasi-autonomous Hamiltonians. Since, as follows from above, the left hand side is quasi-autonomous, the right hand side must be quasi-autonomous too. Moreover, the points where the left hand side achieves its maximum and minimum values are critical points of $G_{\alpha}-F_{\alpha}$ which means that at these points $\mathcal{N}_{t}\left(w_{c}\right)=\nabla S_{t}\left(w_{c}\right)=\nabla F_{\alpha}\left(w_{c}\right)$ is time independent and proves that indeed $H_{t}$ is quasi-autonomous. We remark that the same arguments show that paths with these properties are never unique, as in the definition of $S_{t}$ any function of $t$ such that $f(0)=0$ and $f(1)=1$ can be taken instead of $t$. In our specific case, i.e., $f(t)=t$, in fact autonomous paths are obtained. As a side note, this shows that symplectic maps close to identity always can be generated by autonomous Hamiltonians.

To this end, the conditions of theorem (12) are satisfied, and the Hamilton-Jacobi equation provides a method to compute the distance between symplectic maps. Using (73) in (71), taking norms on both sides of (71), and using the invariance of the oscillation norm under the adjoint action, we obtain

$$
\left\|G_{\alpha}-F_{\alpha}\right\|=\left\|\mu H_{t} \circ \mathcal{M}_{t}\right\|=|\mu||| H_{t}|| .
$$

Integration gives

$$
\left\|G_{\alpha}-F_{\alpha}\right\|=|\mu| \cdot \ell\left\{\phi_{t}\right\}
$$

and according to Siburg's theorem we finally arrive at

$$
\rho(\varphi, \psi)=\frac{1}{|\mu|}\left\|G_{\alpha}-F_{\alpha}\right\| .
$$

In summary, as long as the same type of generating function exists for two compactly supported Hamiltonian maps, the Hofer distance between them can be measured as the oscillation norm of the difference between their generators. From our 
point of view, it is very important that this result holds for any generator type, subject to the existence condition. This is always the case if $\varphi$ and $\psi$ are sufficiently close in the $C^{1}$ topology. However, it is a local result in the sense that it does not provide a way to compute Hofer's distance between any two arbitrary Hamiltonian maps. It is worthwhile to note that for any autonomous Hamiltonian $H$, there exists an $\varepsilon>0$ such that the time one flow of $\varepsilon H$ has only constant fixed points [15]. Also, it automatically follows that flows of quasi-autonomous Hamiltonian systems are geodesics, that is they minimize length on sufficiently small time intervals. It was known from [12] that this is true for autonomous Hamiltonians, and examples of time dependent (quasi-autonomous) Hamiltonians can be found in [14].

\subsection{Some Further Remarks}

In general, Hofer's metric is rather little understood. It determines a kind of $C^{-1}$ topology on $\operatorname{Ham}^{c}\left(\mathbb{R}^{2 n}\right)$, and gives rise to a paradox. Sikorav [21] showed that every one-parameter subgroup of $\operatorname{Ham}^{c}\left(\mathbb{R}^{2 n}\right)$ remains a bounded distance from the identity. So, we could draw the paradoxical conclusion that, according to the main theorem, every point has a flat neighborhood, but in some sense a positive curvature is apparent. In any case, geometry on the group of Hamiltonian symplectomorphisms with respect to Hofer's metric gives rise to a different way of thinking about Hamiltonian dynamics, and we can expect quite some progress in this direction in the near future. The understanding of the global features and properties of Hofer's metric could give insight into the long term properties of Hamiltonian systems, and perhaps provide an exciting method to compute the region of dynamical stability (dynamic aperute in the beam physics jargon) without the time intensive tracking (time consuming numerical integration of large number of sample trajectories). Finally, we mention that there exists another, related metric on $\operatorname{Ham}^{c}\left(\mathbb{R}^{2 n}\right)$, introduced in [22]. However, in [2] and [20] it was proved that they coincide locally on $\mathbb{R}^{2 n}$, so we need not consider it. We use Hofer's metric because it is easier to work with.

Intuitively, Hofer's metric measures what is the minimal Hamiltonian needed, averaged over time, to generate a symplectic map from identity. Actually, Hofer himself calls the distance from the identity of a map the symplectic energy of the map. In view of the KAM theorem for symplectic integration methods $[17,18]$, which roughly says that the behavior of symplectic maps obtained by symplectic integration applied to a integrable system should be close to the integrable system (at least in the region where invariant tori still exist), this provides a strong support 
for our closeness criterion for optimal symplectification based on Hofer's metric, and points out that Hofer's metric is a natural choice.

There is an alternative interpretation of Hofer's metric applied to beam dynamics. Notice that, due to bi-invariance of Hofer's metric we have

$$
\rho(\mathcal{M}, \mathcal{N})=\rho\left(\mathcal{N}^{-1} \circ \mathcal{M}, \mathcal{I}\right) .
$$

Therefore, by computing the distance between symplectic maps in Hofer's metric, actually what is computed is the minimum Hamiltonian needed to generate the initial conditions for $\mathcal{N}$ from the initial conditions for $\mathcal{M}$, such that both $\mathcal{M}$ and $\mathcal{N}$ applied to the respective initial conditions give the same final results. If the result of the norm minimization is small, the two sets of initial conditions are connected by a map close to identity, hence the two sets are close to each other. From a practical point of view this can be considered as not knowing exactly the properties of the injected beam. Such small uncertainties always occur in practice, and to be able to build robust accelerators, one should look for realizations that are not too sensitive to small errors. This is done by considering different error sources, each realization of the errors giving a different symplectic map. The incoming beam quality can be considered one type of such an error effect. Hence, from this viewpoint it does not matter whether $\mathcal{M}$ or $\mathcal{N}$ is used for the simulation of the accelerator at least in the regions of phase space were large-scale chaos is absent.

There are also other interesting consequences of these results, specifically concerning symplectic integration. The only difference between symplectic integration and symplectic tracking is the method for obtaining the truncated generating function. In the case of symplectic tracking, it is obtained from some approximation of $\mathcal{M}$ using (17). In symplectic integration, it is obtained by direct solution of the generalized Hamilton-Jacobi equation (71). Thus, in this case, the linear part $M$ is not known. Therefore, the prescription for optimal symplectic integration is to use Poincaré's generating function and a sufficiently small time step (by this keeping the linear part of the resulting symplectic map as close as possible to identity). Moreover, if the Poincare generator is expanded in a power series in the time step, from the Hamilton-Jacobi equation it follows that, the first order approximation in time is $F_{P}(w)=H(z)$. The corresponding integration method then reduces to the well-known implicit midpoint rule. This is the explanation for the very good reputation that this simple integration method has. 


\section{Summary}

Throughout this paper methods of symplectic geometry and topology have been utilized. The unifying concept behind these methods is the flows of Hamiltonian systems. The systems of interest to us (particle accelerators and other beam physics systems) can be modeled as Hamiltonian systems. The time evolution of these systems (i.e., the flow, or the orbits) can be regarded as curves on the space of Hamiltonian symplectic maps. The geometric properties of these curves with respect to Hofer's metric are deeply related to the dynamics. We exposed and exploited several aspects of this relationship. More precisely, the Hamiltonian system representing the real system is replaced by another Hamiltonian system, and instead of simulating approximately the real system, we track exactly a nearby system, where closeness is measured in Hofer's metric. Then we hope that the perturbations so introduced are small enough that in the light of the KAM theorem most invariant tori, and hence most of the geometric structures, survive, and it leads to a more reliable and faster estimation of the quantities of interest, such as the region of phase space were stable trajectories exist.

The key ingredient was the proof of the main theorem in this paper: locally Hofer's metric between two symplectic maps close in $C^{1}$ can be computed by a norm of the difference of any type of generating function that exist at least locally for both symplectic maps. A generalized Hamilton-Jacobi equation, besides being needed in the proof, is also useful for devising new symplectic integration methods, since it gives the time evolution of the generating functions in terms of the associated Hamiltonian functions.

Finally, while these results have been derived with accelerator physics motivation in mind, their relevance go beyond beam physics, and directly apply to any other weakly nonlinear problem in Hamiltonian dynamics.

\section{References}

[1] Banyaga A., The Structure of Classical Diffeomorphism Groups, Kluwer, Dordrecht, 1997.

[2] Bialy M. and Polterovich L., Geodesics of Hofer's Metric on the Group of Hamiltonian Diffeomorphisms, Duke Math. J. 76 (1994) 273-292.

[3] Dragt A., Lectures on Nonlinear Dynamics and Lie Methods with Applications to Accelerator Physics, Univ. of Maryland preprint, 1998. 
[4] Eliashberg Y. and Polterovich L., Bi-invariant Metrics on the Group of Hamiltonian Diffeomorphisms, Int. J. Math. 4 (1993) 727-738.

[5] Erdelyi B., Global Theory of Extended Generating Functions, Int. J. Pure Applied Math. 33 (2006) 553-578.

[6] Erdelyi B. and Berz M., Optimal Symplectic Approximation of Hamiltonian Flows, Phys. Rev. Lett. 87 (2001) 114302.

[7] Erdelyi B. and Berz M., Local Theory and Applications of Extended Generating Functions, Int. J. Pure Applied Mathematics 11 (2004) 241-282.

[8] Feng K., Wu H., Quin M. and Wang D., Construction of Canonical Difference Schemes for Hamiltonian Formalism via Generating Functions, J. Comp. Math. 7 (1989) 71-96.

[9] Gromov M., Pseudo-Holomorphic Curves in Symplectic Manifolds, Invent. Math. 82 (1985) 307-347.

[10] Hairer E., Lubich C. and Wanner G., Geometric Numerical Integration, Springer, 2002.

[11] Hofer H., On the Topological Properties of Symplectic Maps, Proc. Royal Soc. Edinburgh, Sect. A 115 (1990) 25-38.

[12] Hofer H. and Zehnder E., Symplectic Invariants and Hamiltonian Dynamics, Birkhauser, Basel, 1994.

[13] Lalonde F. and McDuff D., Hofer's $L^{\infty}$ Geometry: Energy and Stability of Hamiltonian Flows. Parts I.\& II., Invent. Math. 122 (1995) 1-33, 35-69.

[14] Long Y., Geodesics in the Compactly Supported Hamiltonian Diffeomorphism Group, Math. Z. 220 (1995) 279-294.

[15] McDuff D. and Salamon D., Introduction to Symplectic Topology, Oxford University Press, New York, 1995.

[16] McDuff D. and Slimowitz J., Hofer-Zehnder Capacity and Length Minimizing Hamiltonian Paths, arXiv:math.SG/0101085.

[17] Shang Z., KAM Theorem of Symplectic Algorithms for Hamiltonian Systems, Numer. Math. 83 (1999) 477-496.

[18] Shang Z., On KAM Theorem for Symplectic Algorithms for Hamiltonian Systems, Ph.D. thesis, CAS Computing Center, Beijing, 1991.

[19] Shashikant M., Berz M. and Erdelyi B., COSY Infinity's EXPO Symplectic Tracking for LHC, Inst. of Physics CS 175 (2004) 299-306.

[20] Siburg K., New Minimal Geodesics on the Group of Symplectic Diffeomorphisms, Calc. Var. and PDEs 3 (1995) 299-309.

[21] Sikorav J., Systemes Hamiltoniens et Topologie Symplectique, ETS, Editrice Pisa, 1990. 
[22] Viterbo C., Symplectic Topology as the Geometry of Generating Functions, Math. Ann. 292 (1992) 685-710.

[23] Weinstein A., Lagrangian Submanifolds and Hamiltonian systems, Ann. Math. 98 (1973) 377-410.

Bela Erdelyi

Department of Physics

Northern Illinois University

DeKalb, IL, 60115

USA

E-mail address: berdelyi@niu.edu 